\title{
Characterizing Heterogeneity of Disease Incidence in a Spatial Hierarchy: A Case Study from a Decade of Observations of Fusarium Head Blight of Wheat
}

\author{
A. B. Kriss, P. A. Paul, and L. V. Madden
}

Department of Plant Pathology, The Ohio State University, Ohio Agricultural Research and Development Center, Wooster 44691. Accepted for publication 8 June 2012.

\begin{abstract}
Kriss, A. B., Paul, P. A., and Madden, L. V. 2012. Characterizing heterogeneity of disease incidence in a spatial hierarchy: A case study from a decade of observations of Fusarium head blight of wheat. Phytopathology 102:867-877.

A multilevel analysis of heterogeneity of disease incidence was conducted based on observations of Fusarium head blight (caused by Fusarium graminearum) in Ohio during the 2002-11 growing seasons. Sampling consisted of counting the number of diseased and healthy wheat spikes per $0.3 \mathrm{~m}$ of row at 10 sites (about $30 \mathrm{~m}$ apart) in a total of 67 to 159 sampled fields in 12 to 32 sampled counties per year. Incidence was then determined as the proportion of diseased spikes at each site. Spatial heterogeneity of incidence among counties, fields within counties, and sites within fields and counties was characterized by fitting a generalized linear mixed model to the data, using a complementary loglog link function, with the assumption that the disease status of spikes

site. Based on the estimated variance terms, there was highly significant spatial heterogeneity among counties and among fields within counties each year; magnitude of the estimated variances was similar for counties and fields. The lowest level of heterogeneity was among sites within fields, and the site variance was either 0 or not significantly greater than 0 in 3 of the 10 years. Based on the variances, the intracluster correlation of disease status of spikes within sites indicated that spikes from the same site were somewhat more likely to share the same disease status relative to spikes from other sites, fields, or counties. The estimated best linear unbiased predictor (EBLUP) for each county was determined, showing large differences across the state in disease incidence (as represented by the link function of the estimated probability that a spike was diseased) but no consistency between years for the different counties. The effects of geographical location, corn and wheat acreage per county, and environmental conditions on the EBLUP for each county were not significant in the majority of years.
\end{abstract} was binomially distributed conditional on the effects of county, field, and
Heterogeneity of plant diseases is the outcome of an array of biological and physical factors that occur at multiple spatial and temporal scales $(33,61)$. Spatial variability at a given time can be at small scales, such as within or among sampling units (sites) within a field, or at larger scales, such as among fields, counties, or states and countries. Additionally, variability can be observed at different temporal scales, such as among assessment times within years, among years, or even among decades.

Several authors have shown how to quantify and interpret the heterogeneity of disease incidence within fields or at higher spatial scales $(17,20,22,33,35)$. The use of discrete probability distributions for overdispersed binary data is especially useful, because a heterogeneity parameter can be estimated to quantify the magnitude of disease aggregation at the scale of the sampling unit or smaller. The $\beta$-binomial distribution has been heavily used for this purpose, partially because one can directly re-express the heterogeneity parameter $(\theta)$ as the intracluster correlation $(\rho)$, a measure of the agreement in disease status of individuals within sampling units (33). As an alternative, Hughes and Samita (22) have proposed the use of the logistic-normal-binomial (LNB) discrete distribution for the same purpose. The LNB can be considered a special case of the marginal distribution that arises when

Corresponding author: L. V. Madden; E-mail address: madden.1@ osu.edu

Any opinions, findings, conclusions, or recommendations expressed in this publication are those of the authors and do not necessarily reflect the view of the United States Department of Agriculture.

http://dx.doi.org/10.1094/PHYTO-11-11-0323

(C) 2012 The American Phytopathological Society fitting a generalized linear mixed model (GLMM) with a logit link, a single random-effect term for the effect of sampling units, and a binomial distribution for number of diseased individuals $(Y)$ conditional on the random effect. Although the $\beta$-binomial distribution is much more commonly used in epidemiology than the LNB, the former cannot be readily expanded to account for multiple sources of variation in a spatial hierarchy (1). In principle, GLMMs can be used for discrete data with any number of spatial scales of variation and can be used to analyze experiments with a wide range of experimental designs $(24,37,52)$. At present, however, there is little evidence regarding the effectiveness of GLMMs for characterizing the heterogeneity of disease incidence at multiple scales in a hierarchy.

Linear mixed models have been effectively used to represent the variability in the incidence of disease of plants or animals, or the counts of organisms, at multiple scales in a spatial hierarchy $(14,45,64)$; however, GLMMs offer several advantages for various data sets (as outlined below). The linear mixed modeling approach is based on the assumption that incidence is a continuous random variable with a normal distribution. However, with GLMMs, nonnormal continuous or discrete data are explicitly incorporated into the model through the use of different distributions in the exponential family. In addition, although one can estimate an intracluster correlation for the agreement of (possibly transformed) mean incidence of the sites within fields using linear mixed models, one cannot estimate the intracluster correlation for disease status of individuals within sampling units, as is possible with GLMMs. Also with GLMMs, it is possible to predict probabilities of specific values of incidence (59) and to drop the assumption that the variance is independent of the mean. Thus, 
there are clear advantages to the use of GLMMs over linear mixed models. Nevertheless, fitting of GLMMs to data is much more computationally demanding compared with the fitting of linear mixed models (2). Until recently, most model-fitting methods for hierarchical GLMMs in commercial software were based on socalled quasi-likelihood or pseudo-likelihood methods (56). With these methods, likelihood-based statistical inference is not generally possible because the likelihood that is calculated is on an arbitrary ("pseudo-data") scale that changes with the random effects in the model. Consequences of this include the inability to directly compare goodness of fit of nested models or to directly determine confidence intervals for variances based on the likelihood (57), the latter being especially important when the objective is to characterize heterogeneity. Major recent advances in statistical computation now make it possible to use maximum likelihood (ML) methods instead of quasi- and pseudo-likelihood methods to fit hierarchical GLMMs directly to data $(2,57)$. Although there are still approximations used to numerically integrate the likelihood function, the full range of likelihood-based inference methods is available to the investigator with these ML methods.

This investigation is part of an overall exploration and evaluation of mixed models for characterizing disease incidence at multiple spatial or temporal scales. The major objective of this article was to use GLMMs to assess the heterogeneity in incidence of Fusarium head blight (FHB) of wheat in Ohio at three scales in a spatial hierarchy (county, field within county, and site within field and county) over a 10-year period. FHB is well known to be highly variable in both space and time, with the variability being related to multiple biological and physical factors $(4,16,30,31,41,66)$; however, the magnitude of FHB heterogeneity has generally not been quantified formally. Through discrete distribution modeling, an analytical protocol for characterizing variability of disease incidence at multiple levels is developed. Secondary objectives were to "estimate" mean incidence of FHB at the county scale using empirical predictions of random effects in the fitted GLMMs for each year, and investigate whether this mean incidence was related to county-aggregated data on environment and cropping information.

\section{MATERIALS AND METHODS}

Data collection. Incidence of FHB on wheat spikes in Ohio wheat fields was obtained through a survey conducted during the 2002-11 growing seasons. Within the state, 12 to 32 counties each year were selected for the survey (Table 1), primarily from the northern and western parts of Ohio, because most of the wheat is grown in these regions. Selection of each county in some years was also influenced by the availability of a county extension educator who was qualified and capable of assessing disease. Within each county, 1 to 15 fields were chosen indiscriminately from across the county (Table 1); field characteristics such as tillage method, cultivar, fungicide use (except for 2011), or other agronomic practices were not considered in field selection. In 2011, we attempted to survey some fields in each county that were sprayed with a fungicide for control of FHB and some fields that were not sprayed with a fungicide. This was the first year with substantial use in Ohio of some of the most effective fungicides labeled for FHB control (P. A. Paul, unpublished data). Most importantly, prior information on FHB disease was not used in selecting fields. Within each field, 10 sites (sampling units) were surveyed in the vast majority of the fields, counties, and years. Occasionally, less than 10 sites per field were surveyed (in about $6 \%$ of the cases). The sites were approximately $30 \mathrm{~m}$ apart and along a main diagonal of the field. As described by Hughes et al. (18), this data collection method within each field is known as cluster sampling.

Each site consisted of $0.3 \mathrm{~m}$ of one wheat row. A measuring "fork" that was $0.3 \mathrm{~m}$ long and attached to a rod was used to isolate only the wheat plants within one row for observations. All wheat tillers within the $0.3 \mathrm{~m}$ were counted to provide the number of spikes at each site $(n)$ and each spike was individually inspected for visual symptoms of FHB. The value of $n$ varied within and among fields, with $80 \%$ of the values being 31 to 62 spikes/

TABLE 1. Characterization of the heterogeneity of Fusarium head blight (FHB) incidence of wheat in Ohio over 10 years based on the fit of equation 3 to the multilevel data, with estimated mean disease incidence (complementary log-log [CLL] scale $[\hat{\mu}]$ and original incidence scale $[\hat{p}]$, with estimated standard error $[S E]$ ) and estimated variances (and $S E$ s) for county $\left(\hat{\sigma}_{C}^{2}\right)$, field within county $\left(\hat{\sigma}_{F}^{2}\right)$, and site within field and county $\left(\hat{\sigma}_{S}^{2}\right)$, with ancillary measures of the intracluster correlation

\begin{tabular}{cccccccccccc}
\hline Year & $\begin{array}{c}\text { Number of } \\
\text { counties }\end{array}$ & $\begin{array}{c}\text { Fields } \\
(\text { mean })^{\mathrm{a}}\end{array}$ & $\hat{\mu}(S E)$ & $\hat{p}(S E)^{\mathrm{b}}$ & $\hat{\sigma}_{C}^{2}(S E)^{\mathrm{c}}$ & $\hat{\sigma}_{F}^{2}(S E)^{\mathrm{d}}$ & $\hat{\sigma}_{S}^{2}(S E)^{\mathrm{e}}$ & $\begin{array}{c}\chi^{2} \\
\left(\hat{\sigma}_{C}^{2}-\hat{\sigma}_{F}^{2}\right)^{\mathrm{f}}\end{array}$ & $\begin{array}{c}\chi^{2} \\
\left(\hat{\sigma}_{F}^{2}-\hat{\sigma}_{S}^{2}\right)^{\mathrm{g}}\end{array}$ & $\hat{\phi}^{\mathrm{h}}$ & $\hat{\rho}^{\mathrm{i}}$ \\
\hline 2002 & 30 & $1-10(5.3)$ & $-3.69(0.14)$ & $0.025(0.003)$ & $0.369(0.145)$ & $0.774(0.125)$ & $0.112(0.023)$ & 3.01 & $70.60^{*}$ & 0.911 & 0.030 \\
2003 & 31 & $1-10(4.8)$ & $-2.89(0.16)$ & $0.054(0.008)$ & $0.647(0.207)$ & $0.515(0.081)$ & $0.075(0.012)$ & 0.38 & $87.26^{*}$ & 0.940 & 0.063 \\
2004 & 19 & $3-15(6.1)$ & $-2.30(0.17)$ & $0.095(0.015)$ & $0.458(0.173)$ & $0.296(0.052)$ & $0.070(0.012)$ & 1.05 & $38.66^{*}$ & 0.914 & 0.073 \\
2005 & 23 & $1-10(5.2)$ & $-5.81(0.21)$ & $0.003(0.001)$ & $0.611(0.300)$ & $0.575(0.173)$ & $0.391(0.129)$ & 0.01 & 0.73 & 0.752 & 0.005 \\
2006 & 17 & $4-11(5.6)$ & $-3.26(0.16)$ & $0.038(0.006)$ & $0.318(0.142)$ & $0.451(0.086)$ & $0(\ldots . j)$ & 0.51 & $\ldots$ & $>0.999$ & 0.028 \\
2007 & 12 & $3-10(5.6)$ & $-5.32(0.39)$ & $0.005(0.002)$ & $1.526(0.895)$ & $0.253(0.117)$ & $0.229(0.105)$ & $6.03^{*}$ & 0.02 & 0.886 & 0.010 \\
2008 & 20 & $2-12(5.1)$ & $-3.58(0.16)$ & $0.027(0.004)$ & $0.399(0.154)$ & $0.357(0.076)$ & $0.020(0.020)$ & 0.06 & $39.29^{*}$ & 0.975 & 0.021 \\
2009 & 21 & $2-6(4.6)$ & $-3.77(0.21)$ & $0.023(0.005)$ & $0.755(0.281)$ & $0.424(0.090)$ & $0.013(0.017)$ & 1.81 & $52.35^{*}$ & 0.989 & 0.026 \\
2010 & 32 & $1-7(4.5)$ & $-1.92(0.17)$ & $0.136(0.021)$ & $0.826(0.227)$ & $0.236(0.037)$ & $0.045(0.006)$ & $15.99^{*}$ & $76.43^{*}$ & 0.959 & 0.131 \\
2011 & 27 & $2-10(5.3)$ & $-2.59(0.18)^{\mathrm{k}}$ & $0.072(0.012)$ & $0.619(0.202)$ & $0.481(0.075)$ & $0.085(0.012)$ & 0.47 & $78.25^{*}$ & 0.929 & 0.060 \\
\hline
\end{tabular}

${ }^{a}$ Range, number of fields per county.

${ }^{\mathrm{b}}$ Estimated SE of the inverse CLL estimate $(\hat{p})$ was based on the delta method.

${ }^{\mathrm{c}}$ In all years, $\hat{\sigma}_{C}^{2}$ was significantly $>0(P<0.005)$ based on likelihood ratio tests.

${ }^{\mathrm{d}}$ In all years, $\hat{\sigma}_{F}^{2}$ was significantly $>0(P<0.005)$ based on likelihood ratio tests.

e $\hat{\sigma}_{S}^{2}$ was significantly $>0(P<0.01)$ in all years except 2006 (where the estimate was 0$), 2008(P=0.153)$, and $2009(P=0.203)$.

${ }^{\mathrm{f}}$ Likelihood-ratio test of the equality of the county and field-within-county variances (* indicates significant at $P<0.05$ ).

g Likelihood-ratio test of the equality of the field-within-county and site-within-field-within-county variances (* indicates significant at $P<0.05$ ).

${ }^{\mathrm{h}}$ An intracluster correlation indicating the degree of similarity of the CLL link of expected probability of disease of sites within fields and counties as calculated from equation 6.

${ }^{i}$ An intracluster correlation indicating the degree of similarity of the disease status of wheat spikes within sites within fields and counties as calculated from equation 8.

j With 0 for the estimated site (i.e., site within field within county) variance in 2006, the corresponding estimated SE is undefined and the test statistic for field variance versus site variance is also undefined. However, because the field variance is $>0$, one can interpret the field variance as being "significantly" larger than the site variance.

${ }^{k}$ Results shown in 2011 are for "no fungicide" use for the control of Fusarium head blight. Results for "fungicide" use are $\hat{\mu}(S E)=-3.20(0.18), \hat{p}(S E)=0.040$ (0.007), and $\hat{\rho}=0.045$. 
site, with an average of 45 . Each person doing the assessments was previously trained on how to recognize symptoms of FHB and how to effectively distinguish between symptoms of FHB and other diseases or injuries in the field. Disease incidence $(Y / n)$ was calculated as the number of spikes with symptoms of FHB $(Y)$ out of the total number of spikes at each site $(n)$. In total, over the 10 years, 1,195 wheat fields and 11,515 sites were sampled; overall, 522,372 wheat spikes were evaluated for disease symptoms.

Data analysis-basic notation. The expected probability of a wheat spike being diseased in any cluster of spikes (such as in a sampling unit or site) is given by $p$. Without consideration of random effects (see below), $p=\mathrm{E}(Y / n)$, where $\mathrm{E}(\bullet)$ represents the expectation of a variable. With random effects, $p$ is considered the expected probability of disease conditional on the random effects (51). For GLMMs, one models a function of $p$, known as the link function, $g(p)$, in relation to any (fixed or random) variables that may be affecting the expected probability. For notational convenience, the symbol $\eta$ is equated to $g(p)$, where $\eta$ is known as the linear predictor. Several choices for the link function are possible, and the logit is the most common for proportion data. However, we used the complementary log-log link $[\operatorname{CLL}(\bullet)]$, given as $\operatorname{CLL}(p)=\ln [-\ln (1-p)]$. This link function was chosen because Paul et al. (47) previously showed that there was a linear relationship between the CLL transformation of FHB spike incidence and the CLL transformation of FHB field severity (the proportion of wheat spikelets with symptoms), known as disease index by many FHB researchers. Hughes et al. (19) also gave a more theoretical foundation for the relation between the CLL of incidence and CLL of severity. Based on the above definitions, one can write the equalities as $g(p)=\operatorname{CLL}(p)=\ln [-\ln (1-p)]=\eta$.

The inverse link function is used to determine $p$ from a given value of $\eta$; the inverse link is given generically by $g^{-1}(\eta)$. For the CLL link, one can then write $p=g^{-1}(\eta)=1-\exp [-\exp (\eta)]$.

With the hierarchical sampling design, we use the subscripts $i$, $j$, and $k$ to label the county, field, and site, respectively. $Y_{i j k}, n_{i j k}$, and $p_{i j k}$ represent the number of the diseased spikes, number of assessed spikes, and the expected conditional probability, respectively, of a spike being diseased for the $k$ th site of the $j$ th field of the $i$ th county. The corresponding link function and linear predictor are given as $g\left(p_{i j k}\right)$ and $\eta_{i j k}$, respectively.

Data analysis-model fitting. Spatial variability was characterized by fitting a GLMM to the incidence data in the spatial hierarchy. In the model, $C_{i}$ represents the effect of the $i$ th county, $F(C)_{i j}$ the effect of the $j$ th field within the $i$ th county, and $S(F C)_{i j k}$ the effect of the $k$ th site within the $j$ th field within the $i$ th county on the linear predictor. County, field, and site were considered to have random effects on the linear predictor; variability was quantified through the variances of the random effects. The random effects were assumed to have normal $(N)$ distributions with means of 0 , and constant variances of $\sigma_{C}^{2}, \sigma_{F}^{2}$, and $\sigma_{S}^{2}$ for county, field within county, and site within field and county, respectively. It was further assumed that the three random effects were independent (32). The model for the linear predictor is written as

$$
g\left(p_{i j k}\right)=\eta_{i j k}=\mu+C_{i}+F(C)_{i j}+S(F C)_{i j k}
$$

where $\mu$ is the overall expected value (mean) on the CLL link scale ("intercept"), and other terms are as defined above.

Using the inverse link function, the expected probability of a spike being diseased conditional on the three random effects of county, field, and site is given (for a CLL link) by

$$
p_{i j k}=g^{-1}\left(\eta_{i j k}\right)=1-\exp \left[-\exp \left(\eta_{i j k}\right)\right]
$$

One can also write

$$
p_{i j k}=\mathrm{E}\left[\left(Y_{i j k} / n_{i j k}\right) \mid C_{i}+F(C)_{i j}+S(F C)_{i j k}\right]
$$

to show that $p_{i j k}$ is an expected value conditional on the $i j k$ th random effects. The number of diseased spikes in the model, $Y_{i j k}$, has a binomial distribution conditional on the random effects; this is equivalent to stating that $Y_{i j k}$ has a conditional binomial distribution. That is, one can write: $Y_{i j k} \sim \operatorname{Bin}\left(p_{i j k}, n_{i j k}\right)$. The so-called marginal distribution of $Y_{i j k}$ has no explicit mathematical form in a hierarchical GLMM (58) but will have a variance larger than a marginal binomial distribution if any of the random-effect variances are larger than 0 . The entire model can be written as

$$
\begin{aligned}
& g\left(p_{i j k}\right)=\eta_{i j k}=\mu+C_{i}+F(C)_{i j}+S(F C)_{i j k} \\
& C_{i} \sim N\left(0, \sigma_{C}^{2}\right) \\
& F(C)_{i j} \sim N\left(0, \sigma_{F}^{2}\right) \\
& S(F C)_{i j k} \sim N\left(0, \sigma_{S}^{2}\right) \\
& p_{i j k}=g^{-1}\left(\eta_{i j k}\right) \\
& Y_{i j k} \sim \operatorname{Bin}\left(p_{i j k}, n_{i j k}\right)
\end{aligned}
$$

As a special case, if there was only one field, there would be no $C$ or $F(C)$ terms, and the single random effect would simply be $S_{k}$; if the link function was the logistic instead of the CLL, then equation 3 would correspond to the logistic-normal-binomial model in Hughes and Samita (22).

Equation 3 was fitted to the FHB incidence data using maximum likelihood with the GLIMMIX procedure in SAS. The integral of the likelihood over the random effects (required for ML estimation) is mathematically intractable for this type of GLMM (38); therefore, it was numerically approximated with a Laplace function in the estimation procedure (using the method=laplace option in GLIMMIX) (49,55). From the fitted model, one obtains estimates of $\mu$ and the three variances, estimated asymptotic standard errors of these terms, and the solutions of the effects of each county $\left(\hat{C}_{i}\right)$, field within county $\left(\widehat{F(C)}_{i j}\right)$, and site within field and county $\left.(\widehat{S(F C})_{i j k}\right)$. In a single year where $\sigma_{S}^{2}$ was estimated at 0 , the model was refitted without the $S(F C)_{i j k}$ term in order to avoid obtaining a nondefinite positive variance-covariance matrix. Because county, field, and site are random effects, the solutions are known as predictors; specifically, the predictors from the model fit are known as estimated best linear unbiased predictors (EBLUPs) (32). Because the expected values of the random effects are 0 , by definition, $\hat{\mu}$ is an estimate of the linear predictor at the means of the random effects, and

$$
\hat{p}=1-\exp (-\exp (\hat{\mu}))
$$

is the estimated (overall) probability of disease at the means of the random effects.

The significance of the random effects was determined with a likelihood-ratio test (55), which consists of a difference in two log-likelihoods (for the model with and without the random-effect term). Under the null hypothesis of no effect, the test statistic has a sampling distribution involving a weighted mixture of $\chi^{2}$ distributions (55). Moreover, likelihood-ratio tests were performed to test the null hypothesis that $\sigma_{C}^{2}=\sigma_{F}^{2}$ and the null hypothesis that $\sigma_{F}^{2}=\sigma_{S}^{2}$. Profile confidence intervals were calculated for each of the random-effect variances. This is the most computationally demanding part of the analysis. All testing of the random effects and determination of the profile confidence intervals were done using the "covtest/cl(type=profile)" statement in the GLIMMIX procedure (55). This procedure automatically determines the weighted combination of $\chi^{2}$ variables for hypothesis testing.

Because fungicide usage was a criterion for selecting fields in 2011, the GLMM was generalized to account for a possible fungicide effect on the overall linear predictor $(\mu)$. In particular, a fixed-effect factor was defined, with two levels $\left(\mu_{i} ; l=1,2\right)$, for the effects on the linear predictor of fungicide or no fungicide use. 
Using $\mu_{l}$ instead of $\mu$, equation 3 was fitted to the data using maximum likelihood. An $F$ test (based on a scaled Wald statistic) was used to determine the effect of fungicide.

County (or field) level predictions. For each of the 10 years, the linear predictor for each county was determined as $\hat{\eta}_{i}=\hat{\mu}+$ $\hat{C}_{i}$. This is the (estimated) linear predictor at the means $(0)$ for the other random effects. Because $\hat{C}_{i}$ is an EBLUP, $\hat{\eta}_{i}$ is also an EBLUP. In terms of inference, this expression for the linear predictor is for a broad inference space (40). Similarly, the broadinference EBLUP for field within county is given as $\hat{\eta}_{i j}=\hat{\mu}+$ $\hat{C}_{i}+\overline{F(C)}_{i j}$. The EBLUP for the expected probability of a spike being diseased for county $i$, conditional on the fields and sites within the county being at their expected values, is given for each county by

$$
\hat{p}_{i}=g^{-1}\left(\hat{\eta}_{i}\right)=g^{-1}\left(\hat{\mu}+\hat{C}_{i}\right)=1-\exp \left(-\exp \left(\hat{\mu}+\hat{C}_{i}\right)\right)
$$

The expression after the last equals sign applies strictly for the CLL link function. Estimated standard errors for EBLUPs obtained from the inverse link were determined using the delta method (55). From use of equation 5, the percentage of counties with probabilities of disease greater than 0.05 ( $>5 \%$ incidence) was also calculated for each year.

EBLUPs for specific fields can be obtained in an analogous manner. That is, the estimated probability of a spike being diseased in field $j$ of county $i$ (when the random site effects are at their expected value [0]) is given by

$$
\hat{p}_{i j}=g^{-1}\left(\hat{\mu}+\hat{C}_{i}+\widehat{F(C)} i j\right)
$$

The GLMM outlined above can be used as one form of a socalled "small area model" (50). When a domain (e.g., area on a map) is considered to be large in a sampling context, direct estimates of the variable of interest (such as simple arithmetic averages of the proportion of diseased spikes) have adequate precision. When a domain is considered small, indirect or synthetic estimates of the variable of interest have greater precision. Because the EBLUP for a domain such as a county is based on all the data (through the model fitting), and not just on the data from the single domain, county-level (or field-level) EBLUPs are considered to be "indirect estimators", "synthetic estimators", or "model-based estimators" (50). In one sense, the EBLUP county values are shrunken (less extreme) compared with samplingdesign-based fixed-effects estimates of disease. This is analogous to the county random-effect values being regressed toward the overall mean based on the magnitude of the random-effect variances (32). For county EBLUPs, the shrinkage toward $\widehat{\mu}$ increases with decreasing $\sigma_{C}^{2}$. This makes the EBLUPs more robust, stable, and precise than direct calculations of incidence. Based on the county EBLUPs, the Spearman rank correlation $(r)$ was calculated between pairs of adjacent years. When there is spatial autocorrelation, the usual calculated (unadjusted) $P$ values for significance of $r$ will be lower than the true $P$ value (9).

The possible effects of geographical location, corn and wheat acreage per county, and environmental conditions on the linear predictor for each county were also examined. This analysis shows whether the county-level variability is due, in part, to these covariables. Geographical location was expressed as the latitude and longitude of the center of each county and was used as a possible effect to see if there were north-south or east-west trends in disease incidence within Ohio. Area of land within each county with corn and wheat, expressed in absolute area (hectares) or as a proportion of the total land in the county, were also considered. The previous year's corn and wheat acreage was investigated because inoculum is produced on infected or infested corn and wheat (46), and increasing abundances of these crops may lead to higher amounts of crop residue and subsequent inoculum pro- duction in the region the following year (27). The current amount (present year) of wheat in the county was considered because it could reflect the level of inoculum in the area. All acreage values were collected from the National Agricultural Statistics Service (www.nass.usda.gov).

Environmental variables were calculated based on data collected from automated weather stations (maintained by the $\mathrm{Na}$ tional Climatic Data Center or the Ohio Agricultural Research and Development Center) in or very near each county. Based on availability of data and previous results (30), two weather variables were calculated based on temperature and precipitation over a 30-day window: total daily precipitation (TotalP) and total daily precipitation when average daily temperature was 15 to $30^{\circ} \mathrm{C}$ (TempP). The two variables were determined for a 30-day window for each county, starting $\approx 1$ week before the estimated start of anthesis.

This covariance analysis was based on fitting an expansion of equation 3 to the data for each year using maximum likelihood. The intercept parameter $\mu$ was expanded to $\mu_{i}$, and expressed as $\mu_{i}=\beta X_{i}$, where $X_{i}$ is one of the covariables for the $i$ th county. Significance was determined using Student $t$ statistics for each estimated $\beta$ parameter.

Intracluster correlations. Measures of the relationship of mean disease incidence (on CLL scale) among sites within fields and counties, and the disease status of wheat spikes within sites (within fields and counties), were made through the use of different versions of the intracluster correlation coefficient (ICC). One first defines the total variance on the CLL-link scale as

$$
\sigma_{\text {Total }}^{2}=\sigma_{C}^{2}+\sigma_{F}^{2}+\sigma_{S}^{2}
$$

The $\phi$ ICC indicates the degree of similarity of the link function of expected conditional probability of disease (i.e., the CLL of mean incidence, a continuous variable) of sites within fields and counties. This ICC is given by

$$
\phi=\frac{\sigma_{C}^{2}+\sigma_{F}^{2}}{\sigma_{C}^{2}+\sigma_{F}^{2}+\sigma_{S}^{2}}=\frac{\sigma_{C}^{2}+\sigma_{F}^{2}}{\sigma_{\text {Total }}^{2}}
$$

$\phi$ can also be thought of as the proportion of the total variance that comes from the differences between the fields and counties. When $\phi$ is near 1 , sites within the same field and county tend to have a very similar level of $\operatorname{CLL}\left(p_{i j k}\right)$ (relative to the total variability over the multiple scales of data in the hierarchy). When $\phi$ is near 0 , sites within the same field and county tend to have very different values of $\operatorname{CLL}\left(p_{i j k}\right)$. Estimates of the variance parameters are substituted for the theoretical values here.

With discrete distributions for disease incidence, interest is often on the intracluster correlation $(\rho)$ of the disease status of individuals within sampling units, which depends on the variability of the number of diseased individuals among the sampling units $(3,11,62)$. With a (marginal) binomial distribution, $\rho=0$, which indicates that the disease status of individuals is independent; with this situation, it is often stated that the disease is random (35). In this investigation, there are three levels of sampling units, with sites nested within fields which are nested within counties. As the degree of heterogeneity among sampling units increases, the probability that the disease status of any two individuals is the same within a site increases; the increase is in direct proportion to $\rho$ (53). The parameter $\rho$ is $>0$ when the $\beta$ binomial distribution (with $\theta>0$ ) or a GLMM with nonzero variance describes the data $(1,34)$. With conditional modeling of discrete data with a nonidentity link function, as done here using GLMMs, there is no exact expression for $\rho$ because the modeling is on different scales. That is, the variance terms are for the variation in the linear predictor $(\eta)$, which is on the scale of a link function (in our case, the CLL link). In contrast, $\rho$ is on the scale of the binary observations for disease status (diseased or healthy). 
As shown in equation 2, $p$ is a nonlinear function of $\eta$, which means that the variance of $p_{i j k}$ can only be approximated based on large-sample theory (26).

There are several different approaches taken to approximate the estimated $\rho$ for hierarchical GLMMs $(3,11,54,62)$. Most of the work has been focused on the two-scale problem, such as multiple sampling units in a single field (62), and we generalize one approach for multiple scales. One method ignores the nonlinear relation between $p$ and $\eta$, and also assumes that the binary observations within sites are a manifestation of an underlying "latent" continuous random variable (12). The other general method explicitly accounts for the nonlinearity of the link function (62), and treats the within-site variable (the individual spike, in our case) as being strictly Bernoulli (a special case of binomial with a sample size of 1) $(54,63)$. We follow this second general approach.

If the variance of $\eta_{i j k}$ is given by $\sigma_{\text {Total }}^{2}$, then, based on the delta method (26), the variance of the random variable $p_{i j k}$ can be approximated by $\operatorname{var}\left(p_{i j k}\right) \approx \sigma_{\text {Total }}^{2} \cdot\left[h^{\prime}(\eta)^{2}\right]$, where $h(\eta)$ is the inverse-link function (i.e., $\left.h[\eta]=g^{-1}[\eta]\right)$, and $h^{\prime}(\eta)$ is the first derivative of the inverse link function (62) with respect to $\eta$. Note that the inverse-link and its first derivative are calculated here at the overall value of the linear predictor (when all random effects are at their expected value of 0 ), which is the $\mu$ term in equation 3 (i.e., when $\eta=\mu$ ). This approximation, which is derived using a Taylor series expansion, is usually accurate unless the variance is very large. For the CLL link, the variance on the incidence scale is specifically

$$
\operatorname{var}\left(p_{i j k}\right) \approx \sigma_{\text {Total }}^{2} \cdot\left[(1-p)^{2} \cdot(-\ln (1-p))^{2}\right]
$$

Here, $p$ is determined from the inverse link of $\mu$ in equation 3 . Following Murray (43), Goldstein et al. (12), and Browne et al. (3), $\rho$ can be written as

$$
\rho \approx \frac{\operatorname{var}\left(p_{i j k}\right)}{\operatorname{var}\left(p_{i j k}\right)+p(1-p)}
$$

where $p$ (no subscript) is the overall probability of disease (inverse link of $\mu$ ), and $p(1-p)$ is the theoretical variance for a Bernoulli distribution. Some authors prefer to call equation 7 a variance partition coefficient. For the specific case of the CLL link function, and three levels to the spatial hierarchy, $\rho$ is approximated as

$$
\rho \approx \frac{\left(\sigma_{C}^{2}+\sigma_{F}^{2}+\sigma_{S}^{2}\right)(1-p)^{2}(-\ln (1-p))^{2}}{\left(\sigma_{C}^{2}+\sigma_{F}^{2}+\sigma_{S}^{2}\right)(1-p)^{2}(-\ln (1-p))^{2}+p(1-p)}
$$

One substitutes the estimate of the variances and $p$ when estimating $\rho$ from a fitted model.

\section{RESULTS}

Heterogeneity at multiple scales. The GLMM in equation 3 was successfully fitted to the FHB incidence data for each of the years using maximum likelihood. Results were for years with as few as 12 counties or as many as 32 counties (Table 1); the average number of fields surveyed per county did not vary much over the decade of disease assessments. Across the 10 years investigated, there was a wide range in the estimated overall mean CLL; $\hat{\mu}$ was lowest in $2005(-5.81)$ and highest in $2010(-1.92)$ (Table 1). In 2011, the estimated mean of fields treated with a fungicide for FHB was significantly lower than the estimated mean of fields that were not treated. Based on the inverse link function (equation 4), estimated statewide mean incidence (more formally: estimated overall probability of a spike being diseased times 100) ranged from $<1 \%$ to $\approx 14 \%$ (Table 1 ).

Even though the estimated statewide mean was very different among the years, spatial heterogeneity at each level in the hierarchy (county, field within county, and site within field and county), as characterized by the three variances in equation 3 , had similar values over all the years (Table 1). This can also be seen by the point estimates and the $95 \%$ profile-likelihood-based confidence intervals for the variances shown in Figure 1. Across the years, the estimated variances at each level had the same order of magnitude, and the confidence intervals had a large degree of overlap for each variance. The width of the confidence intervals for the variances depended, in part, on the number of observations (e.g., number of counties), and generally increased with the magnitude of the point estimates of the variances. As expected, the confidence intervals were not symmetrical, because the sampling distributions of estimated variances are known to be skewed at finite sample sizes (42). There was no apparent trend in the magnitude of the variance estimates with year.

In all years, based on the likelihood-ratio test results, the variances for counties and fields within counties were significantly different from 0 (Table 1), which indicates that heterogeneity at these levels was greater than expected for a binomial (i.e., "random") distribution. In 8 of the 10 years, the point estimate of the county variance $\left(\hat{\sigma}_{C}^{2}\right)$ was similar to the point estimate of the field-within-county variance $\left(\hat{\sigma}_{F}^{2}\right)$. Only in 2007 and 2010 was the point estimate of the variance at the county level significantly higher than at the field-within-county level (Table 1). The magnitude of the point estimates of the variances at the two higher scales was considerably greater than the magnitude of the point estimates of the variances for sites within fields and counties (Fig. 1; Table 1). In no year was the estimated variance for sites within fields and counties greater than the other estimated variances (Table 1). Only in 2007 was $\hat{\sigma}_{S}^{2}$ close to $\hat{\sigma}_{F}^{2}$, and this was a year in which the estimated county variance was much larger than other variances. In 2 of the 10 years (2008 and 2009), the variance among sites within fields and counties was not significantly $>0$; in another year (2006), the site variance was estimated as 0 . For these 3 years, conditional on the field and county, there was only binomial variation in disease incidence within the fields.

County-level predictions. Based on the solution from the fit of equation 3 to the data, the predicted (conditional) probability of disease on the CLL link scale $\left(\widehat{\eta}_{i}=\hat{\mu}+\hat{C}_{i}\right)$ was determined for each county of each year (Table 2 ). Over all years, county-level EBLUPs on the CLL scale were -7.42 to -0.44 . Based on the inverse link (equation 5), the predicted (conditional) probability of disease for the counties was $6 \times 10^{-4}$ to 0.47 . The percentage of counties with an estimated probability of disease $>0.05$ was 0 to $88 \%$. The magnitude of the county EBLUPs within a year (and, hence, the estimated [conditional] probabilities of disease) depended on $\hat{\mu}$ and $\widehat{\sigma}_{C}^{2}$ (the latter determining $\hat{C}_{i}$ ), and the range of EBLUPs within a year was a function of $\widehat{\sigma}_{C}^{2}$ (Table 1). For instance, the range from the minimum to maximum county EBLUP was largest for 2007 and 2010, which had the two largest $\hat{\sigma}_{C}^{2}$ values (1.53 and 0.83, respectively) (Table 1), and was smallest for 2002, 2006, and 2008, which had the three smallest $\hat{\sigma}_{C}^{2}$ values $(0.37,0.32$, and 0.40 , respectively) (Table 1$)$. Because of the nonlinear relation between the conditional probability of disease and the EBLUP, the range in estimated disease probabilities within years had a much more complex relation with the $\widehat{\sigma}_{C}^{2}$ values (Table 1).

In comparison, the field-level EBLUPs $\left(\widehat{\eta}_{i j}=\hat{\mu}+\hat{C}_{i}+\right.$ $\widehat{F(C}_{i j}$ ) were -7.49 to 0.21 and, as required with a nonzero variance for fields within counties, the range was wider in each year compared with the county-level EBLUPs (Table 2). Based on the inverse link, the predicted (conditional) probability of disease for the fields was $6 \times 10^{-4}$ to 0.71 . The percentage of fields with an estimated probability of disease $>0.05$ was 0 to $86 \%$, with the percentage proportional to $\hat{\mu}$ (or $\hat{p}$ ).

The county-level EBLUPs each year were mapped to investigate the spatial arrangement of predictions of FHB across the 
state for the different years (Fig. 2). Results are shown on the CLL-link scale because the model for heterogeneity was fitted on this scale; moreover, if the results were graphed on the incidence (probability) scale (using the inverse link), a different scale of variation would be required on the map for each year (because the variability on a probability scale depends explicitly on the magnitude of the probability) (33). The EBLUPs were also centered at the estimated $\mu$ for each year in the maps (so that only $\widehat{C}_{l}$ is shown) in order to be able to directly compare the countylevel heterogeneity around the expected value (without con- founding by the magnitude of CLL [or it's inverse link]) across years in the same figure. For some years, there was some apparent clustering of counties with similar EBLUP values (or corresponding values of incidence). For instance, in 2010, one can see a large group of counties with predominantly high EBLUPs in the western region of the state, with groups of counties in the north and south with lower EBLUPs (Fig. 2). In most years, there also were situations where there were high $\left(\hat{C}_{i}>1.0\right)$ and low $\left(\hat{C}_{i}<1.0\right)$ EBLUPs in adjacent counties, or a low EBLUP county surrounded by high EBLUP counties, or a high EBLUP county
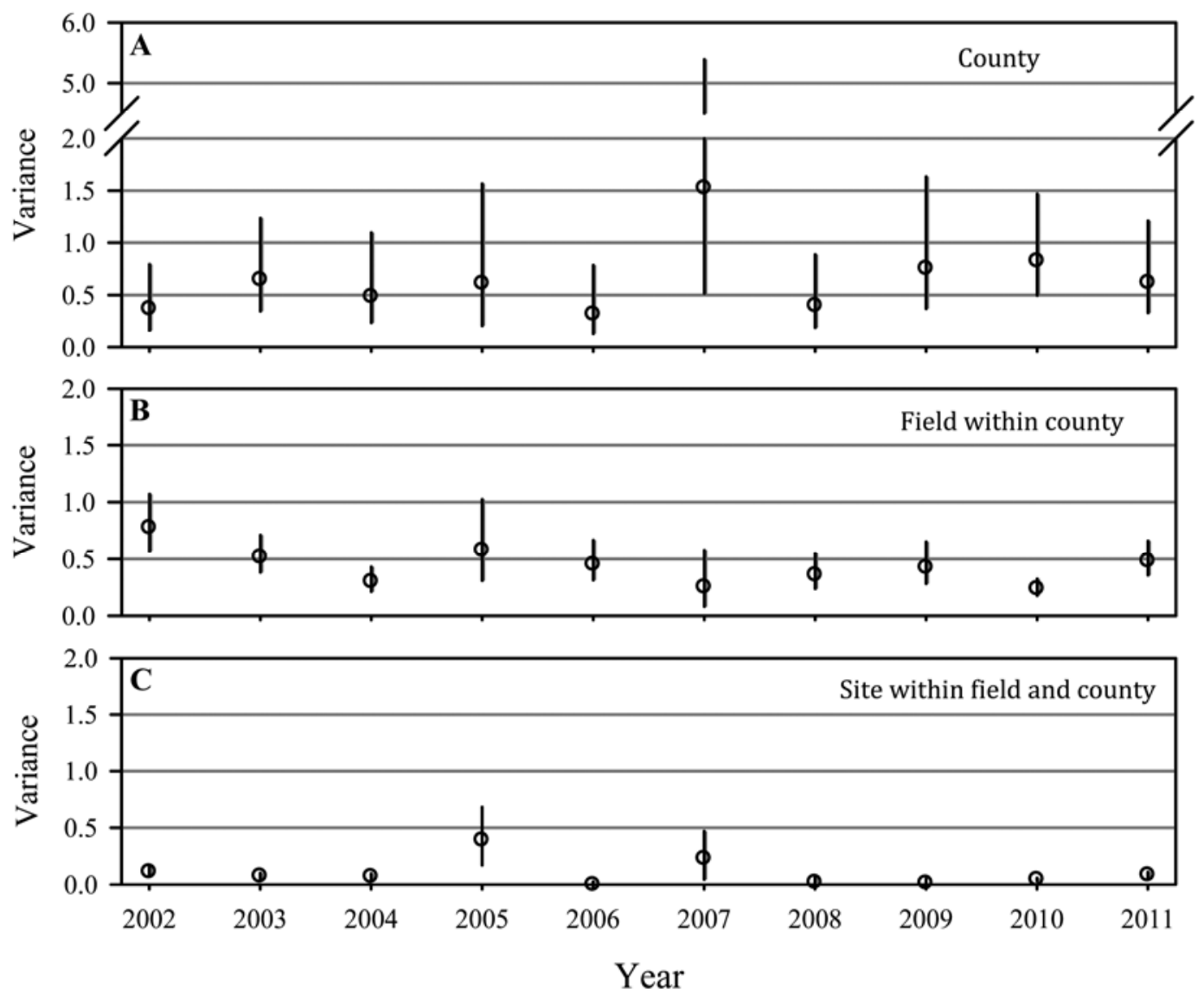

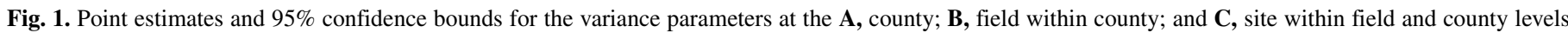
for each year. Standard errors of variance estimates (not directly used in the profile-likelihood-based confidence intervals) are in Table 1.

TABLE 2. Estimated best linear unbiased predictors (EBLUPs) at the county and field levels for the incidence of Fusarium head blight of wheat over 10 years in Ohio (based on the fit of equation 3 to the data) ${ }^{\mathrm{a}}$

\begin{tabular}{|c|c|c|c|c|c|c|}
\hline \multirow[b]{2}{*}{ Year } & \multicolumn{3}{|c|}{ County } & \multicolumn{3}{|c|}{ Field } \\
\hline & $\begin{array}{c}\text { CLL: } \\
{\left[\hat{\mu}+\hat{C}_{i}\right]}\end{array}$ & $\begin{array}{c}\text { Proportion: } \\
\left.\left[g^{-1}\left(\hat{\mu}+\hat{C}_{i}\right)\right]\right]\end{array}$ & $5 \%^{\mathrm{b}}$ & $\left.\begin{array}{c}\text { CLL: } \\
{\left[\hat{\mu}+\hat{C}_{i}+\widehat{F(C)}\right.} \\
i j\end{array}\right]$ & $\begin{array}{c}\text { Proportion: } \\
\left.\left[g^{-1}\left(\hat{\mu}+\hat{C}_{i}+\overline{F(C)}\right)_{i j}\right)\right]\end{array}$ & $5 \%^{\mathrm{b}}$ \\
\hline 2002 & $-4.87 \leftrightarrow-2.80$ & $0.01 \leftrightarrow 0.06$ & $13 \%$ & $-5.95 \leftrightarrow-0.54$ & $3 \times 10^{-3} \leftrightarrow 0.44$ & $25 \%$ \\
\hline 2003 & $-4.55 \leftrightarrow-1.74$ & $0.01 \leftrightarrow 0.16$ & $58 \%$ & $-5.52 \leftrightarrow 0.21$ & $4 \times 10^{-3} \leftrightarrow 0.71$ & $50 \%$ \\
\hline 2004 & $-3.62 \leftrightarrow-0.93$ & $0.03 \leftrightarrow 0.33$ & $84 \%$ & $-4.43 \leftrightarrow-0.10$ & $0.01 \leftrightarrow 0.60$ & $86 \%$ \\
\hline 2005 & $-6.84 \leftrightarrow-4.56$ & $1 \times 10^{-3} \leftrightarrow 0.01$ & $0 \%$ & $-7.10 \leftrightarrow-2.80$ & $8 \times 10^{-4} \leftrightarrow 0.06$ & $1 \%$ \\
\hline 2006 & $-3.83 \leftrightarrow-2.21$ & $0.02 \leftrightarrow 0.10$ & $29 \%$ & $-4.87 \leftrightarrow-1.16$ & $0.01 \leftrightarrow 0.27$ & $43 \%$ \\
\hline 2007 & $-7.42 \leftrightarrow-3.67$ & $6 \times 10^{-4} \leftrightarrow 0.03$ & $0 \%$ & $-7.49 \leftrightarrow-3.26$ & $6 \times 10^{-4} \leftrightarrow 0.04$ & $0 \%$ \\
\hline 2008 & $-4.42 \leftrightarrow-2.46$ & $0.01 \leftrightarrow 0.08$ & $15 \%$ & $-5.34 \leftrightarrow-1.87$ & $5 \times 10^{-3} \leftrightarrow 0.14$ & $29 \%$ \\
\hline 2009 & $-5.30 \leftrightarrow-2.44$ & $5 \times 10^{-3} \leftrightarrow 0.08$ & $14 \%$ & $-6.00 \leftrightarrow-1.16$ & $2 \times 10^{-3} \leftrightarrow 0.27$ & $26 \%$ \\
\hline 2010 & $-4.11 \leftrightarrow-0.44$ & $0.02 \leftrightarrow 0.47$ & $88 \%$ & $-4.55 \leftrightarrow-0.04$ & $0.01 \leftrightarrow 0.62$ & $85 \%$ \\
\hline $2011^{\mathrm{c}}$ & $-3.89 \leftrightarrow-1.46$ & $0.02 \leftrightarrow 0.21$ & $70 \%$ & $-4.19 \leftrightarrow-0.35$ & $0.01 \leftrightarrow 0.51$ & $69 \%$ \\
\hline
\end{tabular}

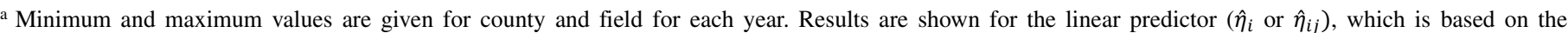
complementary log-log (CLL) link function, and for the proportion scale for disease incidence, which is based on the use of the inverse link function; for example, $\hat{p}_{i}=g^{-1}\left(\hat{\eta}_{i}\right)$. See equation 5 and accompanying text on EBLUPs.

b Percentage of the domains (counties or fields) with an estimated probability of disease incidence (on a percentage scale) $>5 \%$.

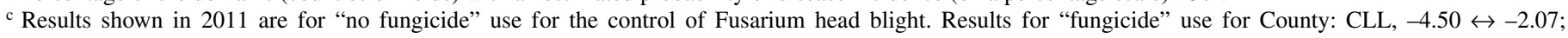
proportion, $0.01 \leftrightarrow 0.12 ; 5 \%, 44 \%$. Results for "fungicide" use for field: CLL, $-5.47 \leftrightarrow-1.17 ;$ proportion, $0.00 \leftrightarrow 0.27 ; 5 \%, 52 \%$. 
surrounded by low EBLUP counties (Fig. 2). As an example, Defiance County in northwestern Ohio in 2011 had a high EBLUP (blue) which was surrounded by low EBLUP counties (red).
There was no consistent temporal pattern to the county $\left(\hat{C}_{i}\right)$ EBLUPs, and the location of the highest or lowest EBLUPs changed from year to year. That is, a county with a large EBLUP in one year could have either a large or small EBLUP the
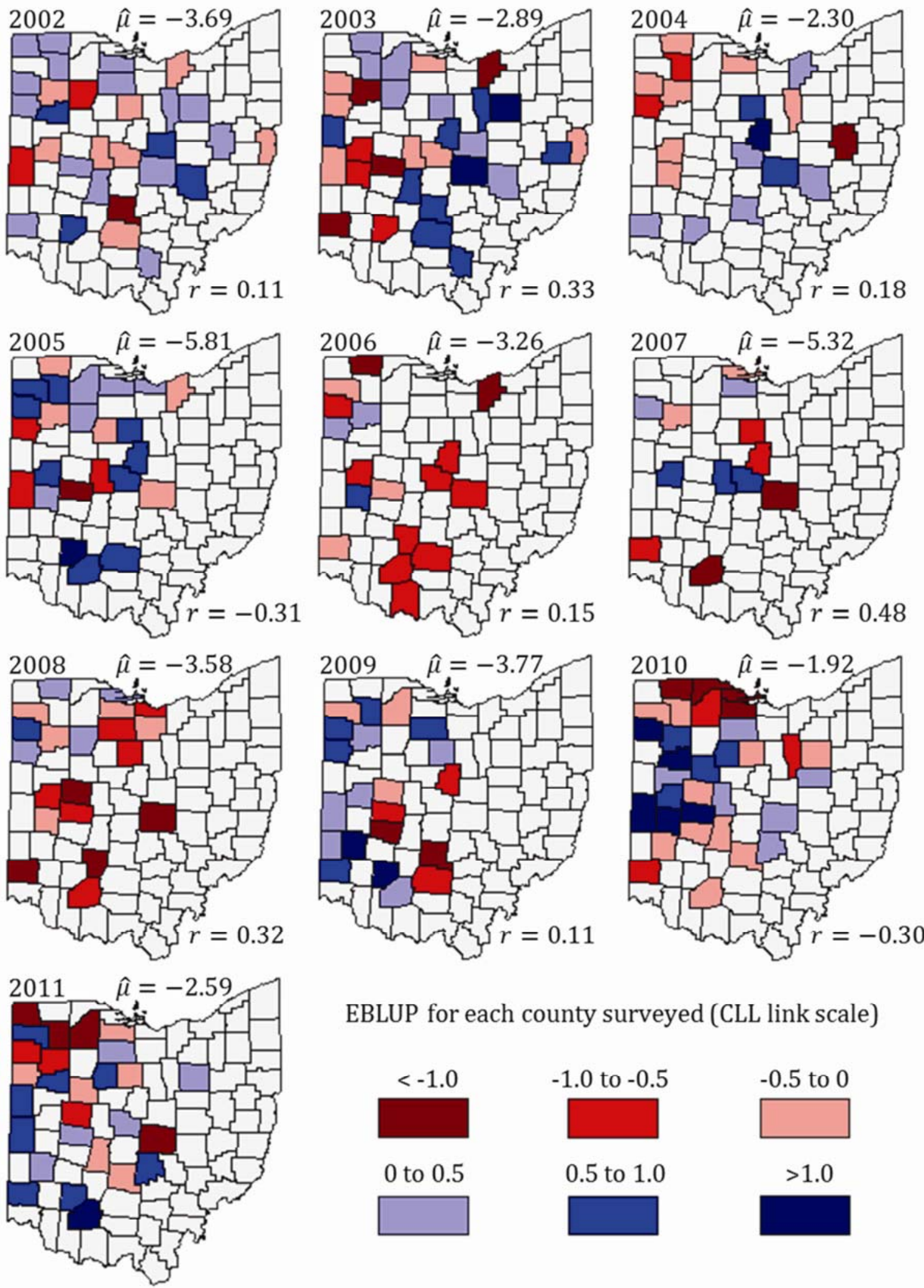

EBLUP for each county surveyed (CLL link scale)

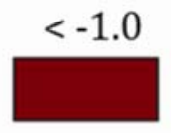

0 to 0.5

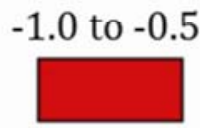

0.5 to 1.0
-0.5 to 0

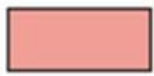

$>1.0$
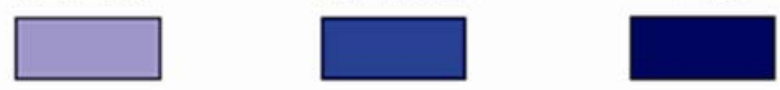

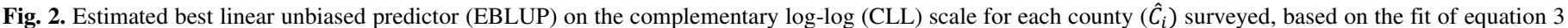

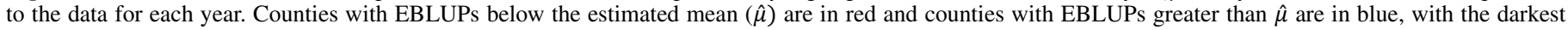

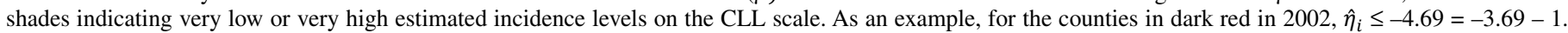

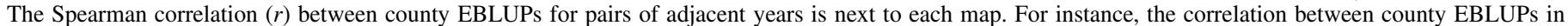

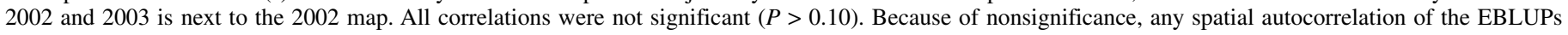
within a year is not relevant for assessing rank correlation. 
following year (Fig. 2). Consider Highland County, the southernmost county surveyed in 2007 to 2011 . The $\hat{C}_{i}$ EBLUP was very negative (i.e., the CLL link and corresponding disease incidence was considerably below the overall mean for the year) in 2007 and 2008 but was moderately high in 2009 , moderately low in 2010 , and very high in 2011. The lack of consistency was confirmed by the Spearman rank correlations $(r)$, which are given on the lower right corner of each map in Figure 2. The correlations were generally all low, and the point estimate could be either positive or negative. None of the estimated rank correlations were significantly different from $0(P>0.10)$.

No county-level covariable had a significant effect in more than three of the years (data not shown). Latitude and longitude were significant in just 2 of the 10 years (but not the same years), and the sign of the parameter estimate $(\hat{\beta})$ was different in the 2 years with significant results. Neither of the two considered environmental variables was ever significant. Corn acreage the previous year was not significant in any year and wheat acreage the previous or present year was not significant in at least $70 \%$ of the years. With wheat acreage per county, the sign of the estimated parameter was not consistent. Thus, given the variability of the linear predictor within counties (Table 2), the county-level covariables were likely to be at too coarse of a measurement scale to account for the variation among counties.

Intracluster correlations. The estimated intracluster correlations for the similarity of disease incidence on the CLL-link scale among sites within fields and counties $(\hat{\phi})$ were very high (Table 1 ) for all years. Most estimates were close to 1 . As indicated by equation 6 , this is because of the very small estimated site-withinfield and county variances $\left(\hat{\sigma}_{S}^{2}\right)$ relative to the county and fieldwithin-county variances for each year. This shows that, in each year, sites within the same field and county tended to have a very similar level of the CLL link of the expected probability of disease. That is, disease incidence for the sites, on a CLL scale, were very similar relative to the variability of disease across fields and counties.

The estimated intracluster correlation of disease status of individuals within the sampling units $(\hat{\rho})$ was generally small. This is partly because $\rho$ is positively related to the magnitude of the variation in expected values of incidence among the sampling units (such as sites) $(17,34,35)$. Because of the low $\hat{\sigma}_{S}^{2}$ values (Table 1 ) in most years, $\hat{\rho}$ (equation 8 ) was generally small. The $\hat{\rho}$ values indicated that, for most years, spikes within the same site were somewhat more likely to share the same disease status relative to spikes from other sites, fields, or counties. For all years except 2005 and 2007, $\hat{\rho}$ was 0.02 to 0.13 . Although these values are not large, they do indicate that the disease status of spikes within sites is not random (as defined by the binomial distribution [where $\rho=0$ ]). The magnitude of $\rho$ is also a complex function of $p$ (see equation 7) (15) but is low at values of $p$ near 0 and 1 for any given value of total variance (on the link scale). In 2005 and 2007 , because the estimated overall probability of disease $(\hat{p})$ was very low, the values of $\hat{\rho}$ were very close to 0 .

\section{DISCUSSION}

The occurrence of disease and magnitude of disease incidence at various locations is directly tied to the heterogeneity of the disease variable (15) over a range of spatial and temporal scales. Several approaches to investigate disease incidence over multiple scales have been reported $(45,48)$, and our specific protocol was built on the use of GLMMs and a conditional binomial distribution to characterize variation in FHB incidence within a spatial hierarchy. GLMMs have been successfully used for characterizing heterogeneity of plant disease incidence when there was only one level to the variation (among sampling units), plus the implicit (conditionally Bernoulli) variation of the binary observations within each sampling unit (21). Expansion to larger scales (multiple fields) typically has been done in a fixed-effects setting, without additional variance terms (22). Others have considered the variation at multiple hierarchical levels in single fields as random effects $(37,48)$ but the interest was in the treatment effects on disease, and the variances were considered nuisance parameters in order to properly estimate the effects of the treatments. GLMMs with multiple random effects have also been successfully used in the analysis of animal disease incidence for several spatial scales $(8,39)$. In these studies, and in the Piepho (48) and Madden et al. (37) reports, quasi- or pseudo-likelihood methods were employed in model fitting, precluding direct inference on the variances based on the magnitude of the likelihood. Here, we were able to use new maximum likelihood computational methods $(49,55)$, with the corresponding likelihood-based procedures for inference, to more directly characterize the variances at three different scales, a primary objective of our investigation. Laplacian approximations to the likelihood may still produce biased parameter estimates (49) but substantial bias typically occurs only when the number of observations at the lowest level in the hierarchy (e.g., number of spikes per site) is very small, with $n=1$ being the extreme (so that each cluster [e.g., site or sampling unit] can only take on one of two values) (25). Laplacian methods are often less biased than pseudo-likelihood methods and the bias is typically very small, with small-tomoderate variances and a large number of observations per cluster at the lowest level in a hierarchy (e.g., number of spikes). With variances typically $<1$ (Table 1 ) and $n$ (more specifically $n_{i j k}$ ) typically being around 45 , bias is not of concern in this investigation.

Even though overall statewide mean disease incidence varied substantially over the 10 years of observations (Table 1), heterogeneity was fairly consistent based on the estimates of the three variances (Fig. 1), with a large degree of overlap of the confidence intervals for each variance across years. There was relatively large and highly significant spatial heterogeneity on the CLL-link scale among counties and fields within counties, and much lower heterogeneity among sites within fields and counties each year. Moreover, point estimates of the county variances tended to be larger than the field-within-county variances in several years, although the county variance was only significantly larger in two of these. This ordering in the variance estimates in a spatial hierarchy has been found in other studies (60). However, the ranking of variances for the levels is not inevitable for spatial data; in fact, the opposite has also been found in the plant and animal sciences. For instance, based on a survey for the incidence of Phomopsis cane and leaf spot of grape and the use of a linear mixed model (with transformed incidence), Nita et al. (45) found that the largest variances were at the lowest spatial scale considered (shoots within vines), with variances decreasing as the scales increased (shoots within vines $\rightarrow$ vines within fields $\rightarrow$ fields within farms $\rightarrow$ farms within regions). In addition, McDermott et al. (39) investigated the incidence of cows with antibodies to the bovine rhinotracheitis virus, and found the greatest variability at the lowest spatial scale evaluated (among farms within areas and districts).

The variability among higher-level domains-fields (and counties)-dominated in the partitioning of the total variance each year in our investigation of FHB. Wilhelm and Jones (65) also indicated a high variability in FHB incidence among fields in their study over 2 years in spring wheat fields in Minnesota. They were able to associate some of the variability to the previous crop grown in the fields but also suggested, as we do here, that there are several field-level factors likely contributing to the heterogeneity between fields. These include planting date, anthesis date, variety, tillage, weed control, and fertilizer use. These field-scale variables were not available or known with our investigation but we expect they vary broadly across the fields and counties. In contrast, we expect less variation in these variables among sites within fields, resulting in a lower among-site variance (relative to 
the others), as found here. Measures on the heterogeneity at the site level have been used to link disease incidence with type of dispersal (airborne or rain-splash) (7).

The variability among sampling units or domains (sites, fields, and counties) can be directly interpreted in terms of the similarity of the response variable within the units, as quantified by one of the intracluster correlations (5). Over each of the years, we found a very high similarity in the (estimated) expected probability of disease on a CLL-link scale for the sites within fields and counties, as measured by $\phi$ (equation 6 ). This was because of the low among-site variance relative to the variances for the higher levels (Table 1). $\phi$ is based on the partitioning of the total variances on the scale of the linear predictor $(\eta)$ and shows the proportion of the higher-level variances relative to the total. This version of the ICC has frequently been estimated for multilevel spatial studies and is applicable whether a linear mixed model for continuous data or a GLMM for discrete or continuous data is used for analysis $(11,63)$. However, only methods designed strictly for discrete data can be used to characterize the similarity of individuals within the lowest level of a hierarchy. Here, we used the $\rho$ ICC of equation 8 , based on the results of the fitted GLMM, to quantify the disease status of wheat spikes within sites within fields and counties. Estimates of $\rho$ were small but nonzero, which is consistent with a high $\phi$. This is partly because, for a fixed $p$, increasing similarity of expected values of disease among the sampling units (and, hence, decreasing site variance) leads to decreasing similarity of individuals within sampling units (20). Although $\rho$ estimates were not large, their magnitude must be considered in the context of the size of the lowest-level sampling units $(\bar{n}$, mean number of spikes per site). For the general situation with discrete data, the observed variance of the number of diseased individuals, $Y$ (suppressing ijk subscripts for convenience) can be written as $\operatorname{var}(Y)=\operatorname{var}(Y)_{(\text {bin })}$.deff, where $\operatorname{var}(Y)_{(b i n)}$ is the variance of $Y$ if it had a binomial distribution, and deff is known as the so-called design effect $(18,28)$, which reflects the overdispersion (extra-binomial variation) of the random variable. With GLMMs involving conditional expected probabilities, the design effect depends on expected disease incidence (17). There is no simple, exact expression for deff with multiple levels in the sampling layout and the use of equation 3 , but it can be approximated as deff $\approx 1+(\bar{n}-1) \cdot \rho$, where $\rho$ is a function of $p$ (equation 8) (5). With a mean of 45 spikes/site, even small $\rho$ values can have a large influence on deff and, hence, on the observed variation. For instance, deff $=2$ is achieved with $\rho \approx$ 0.02 , and $\operatorname{deff}=3$ is achieved with $\rho \approx 0.05$. Thus, the estimated $\rho$ values in our investigation were sufficient to have a clear effect on the degree of overdispersion. It should be noted that others have found that $\rho$ decreases as the size of the sampling unit (i.e., $n$ ) increases $(13,36)$. This is not surprising because, as the size (i.e., $n$ ) of the sampling unit becomes larger, there is more "room" for heterogeneity within the units such as sites.

The estimates of $\rho$ in Table 1 are approximations because the variances used in equation 7 were on the CLL-link scale but $\rho$ is on the scale of the binary variable (63). There is no agreed-upon preferred approximation method for $\rho$ when GLMMs are fitted to data, and limited comparison of approximations do not shed any light on which approach is best (most accurate). We compared several of the published approximations for $\rho(6,43,54)$, and the estimates for most of these were of the same order of magnitude as those given in Table 1 (29) (A. B. Kriss, unpublished data), which means that interpretation was not influenced by the use of equation 7 . The estimated $\rho$ for each year should also be considered a type of composite or "average" correlation of disease status of spikes across the domains in the data set for two reasons. First, $\rho$ depends on the value of $p$ and the site variance (equation 7 or 8 ), and we estimated $\rho$ only for the overall $p$ for each year (based on the inverse link of the estimated $\mu$ ) (Table 1), which is the standard approach for calculating or estimating $\rho$. Second, because the degree of heterogeneity among sites within fields likely depends on the field, the estimated site variance in equation 3 is also a type of composite variance for the linear predictor within fields. If our objective were to characterize the degree of overdispersion in individual fields, as is done often in epidemiology $(7,10)$, we would have needed at least 20 to 30 sites (18) in order to obtain precise site variance estimates for each field. In contrast, our objective was to determine the overall level of heterogeneity for each of the three levels in the hierarchy.

In addition to focusing on the spatial process at the lowest level in the hierarchy, through the ICC, one can use the GLMM-fitting results to focus on the higher-level domains through the empirical predictions (the EBLUPs) for county, field, or even individual site. As discussed in the Materials and Methods section, these EBLUPs ("synthetic estimates" or "small-area estimates") are often more precise (lower mean square error) than other "estimators" of domain means (such as direct estimators [arithmetic averages] obtained with classical survey-sampling methods) because they "borrow strength" from the other domains through the process of model fitting (50). Small-area estimation techniques have been used extensively in many disciplines (23), and have even been used to predict the wheat and corn acreage in individual counties (50), variables we considered in the covariance analysis. Because of their precision, EBLUPs can also be used in "hot-spot" detection for unusually high values of a risktype random variable (44) in a spatial setting. The EBLUPs for counties in Figure 2 show the considerable spatial heterogeneity in FHB incidence over the decade of observations, and hot spots could be identified each year by the EBLUPs $>1$, for instance. However, county EBLUPs were not generally related to geographic location in any given year (based on the analysis of covariance) or to the other county-level covariables in the large majority of years. Moreover, despite the temporal patterns to $\hat{\mu}$ (or corresponding $\hat{p}$ ) over the decade, the high $\hat{C}_{i}$ hot spots (or the low $\hat{C}_{i}$ "cold spots"), relative to $\hat{\mu}$ each year were not found in the same counties over successive years. County borders are obviously based on political decisions, not on agronomic, landscape, or climate factors. However, for policy reasons, there is direct interest in the incidence or risk of disease for these political domains. Because of the high variability of FHB within counties, at approximately the same level as among counties, and the county-scale coarseness of the measurements of the covariables, it is not surprising that there was no clear factor that influenced the among-county heterogeneity. In a future investigation, field-scale measurements of the covariables (local weather, local density of wheat and corn, and so on) could be obtained to determine whether use of these covariables in the model would have an effect on the estimated variances.

In conclusion, there was generally consistent heterogeneity of FHB incidence at multiple spatial scales over the time frame of a decade, with the lowest level of heterogeneity at the lowest scale in the hierarchy. Based on estimated parameters of the fitted GLMM, the intracluster correlation for disease status of individual spikes within sampling sites was low but nonzero for most years. Furthermore, based on the calculated EBLUPs, smallarea estimates of disease incidence for counties could be obtained, although the country rankings varied considerably among years.

\section{ACKNOWLEDGMENTS}

Salaries and research support were provided by state and federal funds to the Ohio Agricultural Research and Development Center (OARDC). This investigation is based upon work supported, in part, by the United States Department of Agriculture (USDA) (agreement number 59-02069-071). Further support was supplied by USDA Cooperative State Research, Education and Extension Service Special Grant 2010-3449321087. This is a cooperative project with the U.S. Wheat \& Barley Scab 
Initiative. We thank the following Ohio State University County Extension Educators, Extension Associates, and cooperators for their contributions to this project: B. Clevenger, R. Amos, G. Arnold, J. Barker, S. Bartels, T. Beck, R. Bender, B. Clevenger, B. Davenport, M. Estadt, S. Evans, S. Foster, J. Grimes, W. Haun, J. Hixson, J. Johnson, A. Kleinschmidt, M. Koenig, G. LaBarge, R. Leeds, E. Lentz, J. Lopshire, T. Mangen, D. Mangione, M. Mechling, D. Mills, S. Mills-Wasniak, T. Nye, S. Prochaska, S. Ruhl, D. Samples, H. Siegrist, K. Simeral, J. Skeeles, J. Stachler, D. Sonnenberg, A. Sundermeier, H. Watters, R. Wells, and G. Wilson. L. V. Madden also thanks K. Garrett for useful discussions nearly a decade ago regarding the potential effects of county-level covariables on disease incidence.

\section{LITERATURE CITED}

1. Anderson, D. A. 1988. Some models for overdispersed binomial data. Aust. J. Stat. 30:125-148.

2. Bolker, B. M., Brooks, M. E., Clark, C. J., Geange, S. W., Poulsen, J. R., Stevens, M. H. H., and White, J.-S. S. 2009. Generalized linear mixed models: A practical guide for ecology and evolution. Trends Ecol. Evol. 24:127-135.

3. Browne, W. J., Subramanian, S. V., Jones, K., and Goldstein, H. 2005. Variance partitioning in multilevel logistic models that exhibit overdispersion. J. R. Stat. Soc. A 168:599-613.

4. Champeil, A. Fourbet, J. F., Doré. T., and Rossignol, L. 2004. Influence of cropping system on Fusarium head blight and mycotoxin levels in winter wheat. Crop Prot. 23:531-537.

5. Collet, D. 1991. Modelling Binary Data. Chapman and Hall, London.

6. Commenges, D., and Jacqmin, H. 1994. The intraclass correlation coefficient: distribution-free definition and test. Biometrics 50:517-526.

7. Del Ponte, E. M., Shah, M., and Bergstrom, G. C. 2003. Spatial patterns of Fusarium head blight in New York wheat fields suggests role of airborne inoculum. Plant Health Progress. Online publication. doi:10.1094/PHP-2003-0418-01-RS

8. Dohoo, I. R., Tillard, E., Stryhn, H., and Faye, B. 2001. The use of multilevel models to evaluate sources of variation in reproductive performance in dairy cattle in Reunion Island. Prev. Vet. Med. 50:127144.

9. Dutilleul, P., Clifford, P., Richardson, S., and Hemon, D. 1993. Modifying the $t$ test for assessing the correlation between two spatial processes. Biometrics 49:305-314.

10. El-Allaf, S. M., Madden, L. V., and Lipps, P. E. 2002. Spatial aspects of Fusarium head blight epidemics on wheat in Ohio. Pages 148-150 in: Proc. 2002 Natl. Fusarium Head Blight Forum. Erlanger, KY.

11. Eldridge, S. M., Ukoumunne, O. C., and Carlin, J. B. 2009. The intracluster correlation coefficient in cluster randomized trials: A review of definitions. Int. Stat. Rev. 77:378-394.

12. Goldstein, H., Browne, W., and Rasbash, J. 2002. Partitioning variation in multilevel models. Underst. Stat. 1:223-231.

13. Gosme, M., Willocquet, L., and Lucas, P. 2007. Size, shape and intensity of aggregation of take-all disease during natural epidemics in second wheat crops. Plant Pathol. 56:87-96.

14. Gray, B. R., Haro, R. J., Rogala, J. T., and Sauer, J. S. 2005. Modelling habitat associations with fingernail clam (family: Sphaeriidae) counts at multiple spatial scales using hierarchical count models. Freshwater Biol. 50:715-729.

15. Gulliford, M. C., Adams, G., Ukoumunne, O. C., Latinovic, R., Chinn, S., and Campbell, M. J. 2005. Intraclass correlation coefficient and outcome prevalence are associated in clustered binary data. J. Clin. Epidemiol. 58:246-251.

16. Hollingsworth, C. R., Motteberg, C. D., Wiersma, J. V., and Atkinson, L. M. 2008. Agronomic and economic responses of spring wheat to management of Fusarium head blight. Plant Dis. 92:1339-1348.

17. Hughes, G., and Madden, L. V. 1995. Some methods allowing for aggregated patterns of disease incidence in the analysis of data from designed experiments. Plant Pathol. 44:927-943.

18. Hughes, G., Madden, L. V., and Munkvold, G. P. 1996. Cluster sampling for disease incidence data. Phytopathology 86:132-137.

19. Hughes, G., McRoberts, N., and Madden, L. V. 2004. Daamen's incidence-severity relationship revisited. Eur. J. Plant Pathol. 110:759-761.

20. Hughes, G., McRoberts, N., Madden, L. V., and Gottwald, T. R. 1997. Relationships between disease incidence at two levels in a spatial hierarchy. Phytopathology 87:542-550.

21. Hughes, G., Munkvold, G. P., and Samita, S. 1998. Application of the logistic-normal-binomial distribution to the analysis of Eutypa dieback disease incidence. Int. J. Pest Manage. 44:35-42.

22. Hughes, G., and Samita, S. 1998. Analysis of patterns of pineapple mealybug wilt disease in Sri Lanka. Plant Dis. 82:885-890.

23. Jiang, J., and Lahiri, P. 2006. Mixed model prediction and small area estimation. Test 15:1-96.

24. Jirak-Peterson, J. C., and Esker, P. D. 2011. Tillage, crop rotation, and hybrid effects on residue and corn anthracnose occurrence in Wisconsin. Plant Dis. 95:601-610.

25. Joe, H. 2008. Accuracy of Laplace approximation for discrete response mixed models. Comput. Stat. Data Anal. 52:5066-5074.

26. Keener, R. W. 2010. Large-sample theory. Pages 129-149 in: Theoretical Statistics. Springer, New York.

27. Keller, M. D., Thomason, W. E., and Schmale, D. G., III. 2011. The spread of a released clone of Gibberella zeae from different amounts of infested corn residue. Plant Dis. 95:1458-1464.

28. Kish, L. 1965. Survey Sampling. John Wiley \& Sons, New York.

29. Kriss, A. B. 2011. Pages 226-234 in: The role of environmental, temporal, and spatial scale on the heterogeneity of Fusarium head blight of wheat. Appendix B. Intra-cluster correlations for binary data in hierarchical random effects models. Ph.D. dissertation, The Ohio State University.

30. Kriss, A. B., Paul, P. A., and Madden, L. V. 2010. Relationship between yearly fluctuations in Fusarium head blight intensity and environmental variables: a window-pane analysis. Phytopathology 100:784-797.

31. Kriss, A. B., Paul, P. A., and Madden, L. V. 2012. Variability in Fusarium head blight epidemics in relation to global climate fluctuations as represented by the El Niño Southern Oscillation and other atmospheric patterns. Phytopathology 102:55-64.

32. Littell, R. C., Milliken, G. A., Stroup, W. W., Wolfinger, R. D., and Schabenberger, O. 2006. SAS for Mixed Models, Second Edition. SAS Institute Inc., Cary, NC.

33. Madden, L. V., and Hughes, G. 1995. Plant disease incidence: Distributions, heterogeneity, and temporal analysis. Annu. Rev. Phytopathol. 33:529564.

34. Madden, L. V., and Hughes, G. 1999. Sampling for plant disease incidence. Phytopathology 89:1088-1103.

35. Madden, L. V., Hughes, G., and van den Bosch, F. 2007. The Study of Plant Disease Epidemics. American Phytopathological Society, St. Paul, MN.

36. Madden, L. V., Nault, L. R., Murral, D. J., and Apelt, M. R. 1995. Spatial pattern analysis of the incidence of aster yellows disease in lettuce. Res. Popul. Ecol. 37:279-289.

37. Madden, L. V., Turechek, W. W., and Nita, M. 2002. Evaluation of generalized linear mixed models for analyzing disease incidence data obtained in designed experiments. Plant Dis. 86:316-325.

38. McCulloch, C. E. 2000. Generalized linear models. J. Am. Stat. Assoc. 95:1320-1324.

39. McDermott, J. J., Kadohira, M., O'Callaghan, C. J., and Shoukri, M. M. 1997. A comparison of different models for assessing variations in the sero-prevalence of infectious bovine rhinotracheitis by farm, area and district in Kenya. Prev. Vet. Med. 32:219-234.

40. McLean, R. A, Sanders, W. L., and Stroup, W. W. 1991. A unified approach to mixed linear models. Am. Stat. 45:54-64.

41. McMullen, M., Jones, R., and Gallenberg, D. 1997. Scab of wheat and barley: A re-emerging disease of devastating impact. Plant Dis. 81:13401348.

42. Meyer, K., and Hill, W. G. 1992. Approximation of sampling variances and confidence intervals for maximum likelihood estimates of variance components. J. Anim. Breed. Genet. 109:264-280.

43. Murray, D. M. 1998. Design and Analysis of Group-Randomized Trials. Oxford University Press, New York.

44. Nelson, T. A., and Boots, B. Detecting spatial hot spots in landscape ecology. Ecography 31:556-566.

45. Nita, M., Ellis, M. A., and Madden, L. V. 2008. Variation in disease incidence of Phomopsis cane and leaf spot of grape in commercial vineyards in Ohio. Plant Dis. 92:1053-1061.

46. Osborne, L. E., and Stein, J. M. 2007. Epidemiology of Fusarium head blight on small-grain cereals. Int. J. Food Microbiol. 119:103-108.

47. Paul, P. A., El-Allaf, S. Lipps, P. E., and Madden, L. V. 2005. Relationships between incidence and severity of Fusarium head blight on winter wheat in Ohio. Phytopathology 95:1049-1060.

48. Piepho, H.-P. 1999. Analysing disease incidence data from designed experiments by generalized linear mixed models. Plant Pathol. 48:668-674.

49. Pinheiro, J. C., and Chao, E. C. 2006. Efficient Laplacian and adaptive Gaussian quadrature algorithms for multilevel generalized linear mixed models. J. Comput. Graph. Stat. 15:58-81.

50. Rao, J. N. K. 2003. Small Area Estimation. John Wiley \& Sons, New York.

51. Raudenbush, S. W., Yang, M.-L., and Yosef, M. 2000. Maximum likelihood for generalized linear models with nested random effects via high-order, multivariate Laplace approximation. J. Comput. Graph. Stat. 9:141-157.

52. Redinbaugh, M. G., Molineros, J. E., Vacha, J., Berry, S. A., Hammond, R. B., Madden, L. V., and Dorrance, A. E. 2010. Bean pod mottle virus spread in insect-feeding-resistant soybean. Plant Dis. 94:265-270. 
53. Ridout, M. S., Demétrio, C. G. B., and Firth, D. 1999. Estimating intraclass correlation for binary data. Biometrics 55:137-148.

54. Sashegyi, A. I., Brown, K. S., and Farrell, P. J. 2001. On the correspondence between population-averaged models and a class of clusterspecific models for correlated binary data. Stat. Prob. Lett. 52:135-144.

55. SAS Institute Inc. 2008. SAS/STAT 9.2 User's Guide: The GLIMMIX Procedure (Book Excerpt). SAS Institute Inc., Cary, NC.

56. Schabenberger, O. 2005. Introducing the GLIMMIX procedure for generalized linear mixed models. SAS Users Group International (SUGI) 30. Paper 196-30. SAS Institute Inc., Cary, NC.

57. Schabenberger, O. 2007. Growing up fast: SAS 9.2 enhancements to the GLIMMIX procedure. SAS Global Forum 2007. Paper 177. SAS Institute Inc., Cary, NC.

58. Schabenberger, O., and Pierce, F. J. 2002. Contemporary Statistical Models for the Plant and Soil Sciences. CRC Press, Boca Raton, FL.

59. Skrondal, A., and Rabe-Hesketh, S. 2003. Some applications of generalized linear latent and mixed models in epidemiology: Repeated measures, measurement error and multilevel modeling. Nor. Epidemiol. $13: 265-278$.

60. Suomela, J. 1996. Within-tree variability of mountain birch leaves causes variation in performance for Epirrita autumnata larvae. Vegetatio 127:77-83.
61. Turechek, W. W., and Madden, L. V. 2001. Effect of scale on plant disease incidence and heterogeneity in a spatial hierarchy. Ecol. Model. 144:7795.

62. Turner, R. M., Omar, R. Z., and Thompson, S. G. 2001. Bayesian methods of analysis for cluster randomized trials with binary outcome data. Stat. Med. 20:453-472.

63. Turner, R. M., Omar, R. Z., and Thompson, S. G. 2006. Constructing intervals for the intracluster correlation coefficient using Bayesian modeling, and application in cluster randomized trials. Stat. Med. 25:14431456.

64. Werneck, G. L., Costa, C. H. N., Walker, A. M., David, J. R., Wand, M., and Maguire, J. H. 2007. Multilevel modeling of the incidence of visceral leishmaniasis in Teresina, Brazil. Epidemiol. Infect. 135:195-201.

65. Wilhelm, K. P., and Jones, R. K. 2005. Meso- and microscale patterns of Fusarium head blight in spring wheat fields in Minnesota. Plant Dis. 89:474-479.

66. Xu, X.-M., Parry, D. W., Nicholson, P., Thomsett, M. A., Simpson, D. Edwards, S. G., Cooke, B. M., Doohan, F. M., Monaghan, S., Moretti, A., Tocco, G., Mule, G., Hornok, L., Béki, E., Tatnell, J., and Ritieni, A. 2008. Within-field variability of Fusarium head blight pathogens and their associated mycotoxins. Eur. J. Plant Pathol. 120:21-34. 